\title{
Attitude and Altitude Control of Quadcopter Maneuvers using Neural Network-Based Direct Inverse Control
}

\author{
M. Ary Heryanto ${ }^{1}$, Benyamin Kusumoputro ${ }^{2 *}$ \\ ${ }^{1}$ Department of Electrical Engineering, Faculty of Engineering, Universitas Dian Nuswantoro, Semarang \\ 50131, Indonesia \\ ${ }^{2}$ Department of Electrical Engineering, Faculty of Engineering, Universitas Indonesia, Kampus UI Depok, \\ Depok 16424, Indonesia
}

\begin{abstract}
In this study, a neural network-based direct inverse control (DIC) approach was developed and simulated to control various maneuvers of an unmanned aerial vehicle (UAV) quadcopter. The aim was to propose an inner loop control algorithm for UAV quadcopter maneuvers using a neural network-based DIC system. The appropriate connection weights of neurons in the controller were determined through a backpropagation learning algorithm using real quadcopter maneuver flight data. The neural network-based DIC was trained and then tested using a trajectory dataset different from the training dataset. The experimental results showed that the neural network-based DIC could follow the maneuvers of the testing trajectory dataset with excellent performance, as indicated by an overall mean squared error (MSE) of 1.461 and attitude MSEs of 3.104 for roll, 0.889 for pitch, 1.834 for yaw and 0.018 for altitude. These results indicate that the proposed artificial neural network-based DIC can be used to control the attitude and altitude of the quadcopter during maneuvers.
\end{abstract}

Keywords: Backpropagation; Direct inverse control; Maneuver; Neural Network; Quadcopter

\section{Introduction}

Unmanned aerial vehicle (UAV) research has grown rapidly in recent years in both the civilian and military sectors (Duan et al., 2010; Gandhi and Ghosal, 2018; Krishnan et al., 2018; Nenni et al., 2020). As multirotor drones, UAV quadcopters have several advantages, including the ability to perform vertical takeoff and landing, high maneuverability, and simple mechanical structures. Quadcopters are under-actuated, with strong coupling and highly nonlinear systems (Gheorghita et al., 2015; Wang et al., 2016); therefore, controlling them is a considerable challenge.

UAV quadcopters are increasingly attracting the attention of researchers, and developing an autonomous quadcopter control method is of central importance. Numerous studies have been conducted to address the issue of quadcopter control. Proportional integral derivative (PID), LQR, backstepping, and sliding mode control are the most widely used methods (Argentim et al., 2013; Tripathi et al., 2015; Wang et al., 2016; Najm and Ibraheem, 2019; Nguyen et al., 2019).

Moreover, neural network (NN)-based control systems have been developed with

*Corresponding author's email: nynykusumo@yahoo.com,, Tel.: +62-21-7270078; Fax: +62-21-7270077 doi: 10.14716/ijtech.v12i4.3928 
decent performance (Anuradha et al., 2009; Xianglei et al., 2011; Suprapto et al., 2017; Muliadi \& Kusumoputro, 2018; Yuning et al., 2019; Mahadika et al., 2020).

Like other aerial vehicles, quadcopters have abilities of six degrees of freedom-pitch, roll, yaw, and $x, y, z$-but only four basic movements are generated directly-namely, roll, pitch, yaw, and thrust (Wang et al., 2016). Roll and pitch are related to changes in the quadcopter's position on the $x$ and $y$ axes, and yaw is related to rotational motion on the $z$ axis. Roll, pitch, and yaw produce attitude movement, while thrust is related to changes in altitude movement. Since the quadcopter movement depends entirely on these four basic motions, it is extremely important to maintain stable attitude (pitch, roll, and yaw) and altitude control conditions, especially during maneuvers.

Our research is based on a cross configuration of a quadcopter constructed in the Computational Intelligence and Intelligent Systems Laboratory, Universitas Indonesia. We previously also developed an NN-DIC scheme as a control system and optimized it to easily control the hovering state of the quadcopter (Heryanto et al., 2015). Our results showed that the optimized NN-DIC system improved the controller's performance, as indicated by a faster settling time compared with that of a non-optimized NN-DIC method. We also confirmed in subsequent experiments (Heryanto et al., 2017) that the NN-DIC system can maintain the quadcopter's attitude and altitude in a simple UAV simulation flight with reasonable errors. Based on these results, in this study, we aimed to investigate the response of attitude and altitude control using our developed NN-DIC method on a maneuvering trajectory flight of a quadcopter.

The rest of this paper is organized as follows. Section 2 explains the structure and dynamics of the quadcopter. Section 3 describes the NN-DIC strategy and the data acquisition method. Section 4 presents the simulation results of the experiment. Section 5 concludes the paper.

\section{Quadcopter Basic Movement}

All quadcopters have the same mechanical design, consisting of four motors located at each corner, coupled with fixed pitch propellers generating equal thrust. The motors are divided into two pairs: one pair, located at the front and rear, rotates clockwise, and the other pair, located on the right and left, rotates counterclockwise to cancel the rotational torque (Figure 1) while allowing the quadcopter to move by changing the propeller velocities (M1, M2, M3, and M4).

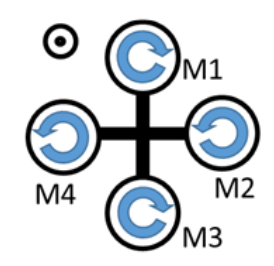

(a) Throttle, up/down

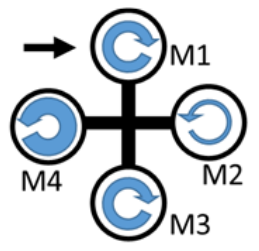

(b) Roll, right

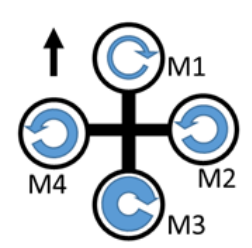

(c) Pitch, forward

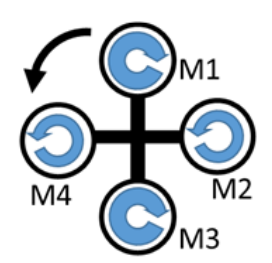

(d) Yaw, left rotation

Figure 1 The basic movement of the quadcopter

The quadcopter has four basic movements that are directly controlled by the four rotors: thrust, roll, pitch, and yaw. As shown in Figure $1 \mathrm{a}$, the $\mathrm{U}_{1}$ force $(\mathrm{N})$ generates an upand-down movement by maintaining all the propellers at the same speed. Changing the velocities of M2 and M4 (Figure $1 \mathrm{~b}$ ) generates the $U_{2}$ force $(\mathrm{Nm})$, which affects the attitude of the quadcopter, namely roll. When the velocity of M4 $\left(\Omega_{4}\right)$ is higher than that of M2 $\left(\Omega_{2}\right)$, the quadcopter rolls right and eventually moves to the right. When the velocity of M4 is lower than that of M2, the quadcopter moves to the left. 
Likewise, pitch conditions occur when the velocities of M1 and M3 are different (Figure $1 \mathrm{c})$, and the $\mathrm{U}_{3}$ force $(\mathrm{Nm})$ is determined by this difference in velocities. When the velocity of M1 $\left(\Omega_{1}\right)$ is lower than that of M3 $\left(\Omega_{3}\right)$, the attitude of the quadcopter is pitched down, and the quadcopter moves forward. Conversely, when the velocity of M1 is higher than that of M3, the attitude of the quadcopter is pitched up, and the quadcopter moves backward. The last movement (i.e., yaw) is rotation on the quadcopter shaft (Figure 1d). Yaw produces the $\mathrm{U}_{4}$ force $(\mathrm{Nm})$ when the two propeller pairs have different velocities. When the velocity of the M1-M3 pair is higher than that of the M2-M4 pair, the quadcopter rotates left. Conversely, when the velocity of the M1-M3 pair is lower than that of the M2-M4 pair, the quadcopter rotates right.

Researchers have modeled quadcopters using various types of structures and configurations (Wang et al., 2016; Alkamachi and Erçelebi, 2017; Najm et al., 2020). The most frequently used model is the cross configuration. As quadcopters have nonlinear, cross-coupling, and under-actuated characteristics, it is difficult to develop a model that closely simulates a real quadcopter. Numerous assumptions have been adopted to simplify quadcopter models, such as a symmetrical and rigid quadcopter structure, rigid propellers, thrust and drag forces proportional to the square of the propellers' rotation speed, and symmetrical quadcopter mass distribution (Bresciani, 2008).

As previously noted, the velocities of M1, M2, M3, and M4 influence the thrust, roll, pitch, and yaw forces. The equation describing the relationship between the basic movements and the forces is as follows:

$$
\begin{aligned}
& U_{1}=b\left(\Omega_{1}^{2}+\Omega_{2}^{2}+\Omega_{3}^{2}+\Omega_{4}^{2}\right) \\
& U_{2}=l b\left(-\Omega_{2}^{2}+\Omega_{4}^{2}\right) \\
& U_{3}=l b\left(-\Omega_{1}^{2}+\Omega_{3}^{2}\right) \\
& U_{4}=d\left(-\Omega_{1}^{2}+\Omega_{2}^{2}-\Omega_{3}^{2}+\Omega_{4}^{2}\right)
\end{aligned}
$$

where $U_{1}$ is thrust, $U_{2}$ is roll, $U_{3}$ is pitch, $U_{4}$ is yaw, $\Omega_{\mathrm{n}}$ denotes the propeller velocities ( $\mathrm{n}=$ $1,2,3$, and 4$), b$ is the thrust coefficient $\left(\mathrm{Ns}^{2}\right), d$ is the drag coefficient $\left(\mathrm{Nms}^{2}\right), l$ is the distance between the center of the propeller and the center of the quadcopter $(\mathrm{m})$, and $\Omega$ is the sum of the propeller velocities as calculated by Equation 2. The quadcopter dynamics used in attitude and altitude control are calculated by Equation 3.

$$
\begin{aligned}
& \Omega=-\Omega_{1}+\Omega_{2}-\Omega_{3}+\Omega_{4} \\
& \Omega_{1}=-g+(\cos \theta \cos \varphi) \frac{U_{1}}{m} \\
& \Omega_{2}=\frac{U_{2}}{I_{X X}} \\
& \Omega_{3}=\frac{U_{3}}{I_{Y Y}} \\
& \Omega_{4}=\frac{U_{4}}{I_{Z Z}}
\end{aligned}
$$

where $\Omega_{1}$ is the quadcopter's linear acceleration along the $z$ axis $\left(\mathrm{ms}^{-2}\right), g$ is gravity acceleration ( $\mathrm{ms}^{-2}$ ), $\theta$ is the quadcopter's angular position around the $y$ axis (rad); $\phi$ is the quadcopter's angular position around the $x$ axis (rad); $m$ is the quadcopter's mass (kg), $\Omega_{2}$ is the quadcopter's angular acceleration around the $x$ axis ( $\mathrm{rad} \mathrm{s}^{-2}$ ), $\Omega_{3}$ is the quadcopter's angular acceleration around the $y$ axis $\left(\mathrm{rad} \mathrm{s}^{-2}\right), \Omega_{4}$ is the quadcopter's angular acceleration around the $z$ axis $\left(\mathrm{rad} \mathrm{s}^{-2}\right)$, and $I_{X X}, I_{Y Y}$, and $I_{Z Z}$ are the body's moment of inertia around the $x, y$, and $z$ axes, respectively $\left(\mathrm{Nms}^{2}\right)$. 


\section{Neural Network-Based Direct Inverse Control}

\subsection{Control Strategy}

As shown in Figure 2, the quadcopter control is generally divided into two partsattitude and altitude control (inner loop) and position control (outer loop) -with $x, y$, and $z$ being the target position coordinate, $\hat{x}, \hat{y}$, and $\hat{z}$ being the real position coordinate, $\phi$, $\theta$, and $\psi$ being the target angular position, and $\hat{\phi}, \hat{\theta}$, and $\hat{\psi}$ being the real angular position. The control value is the pulse-width modulation (PWM) value of the Remote Control Pulse Standard (RCPS), which is proportional to the propeller velocity. The focus of this research was on developing an inner loop control system under maneuver conditions. Referring to Equations 1 to 3, the set points on the inner loop control are attitude $(\phi, \theta$, and $\psi$ ) and altitude (z), whereas the inner loop control output is the propellers' velocities.

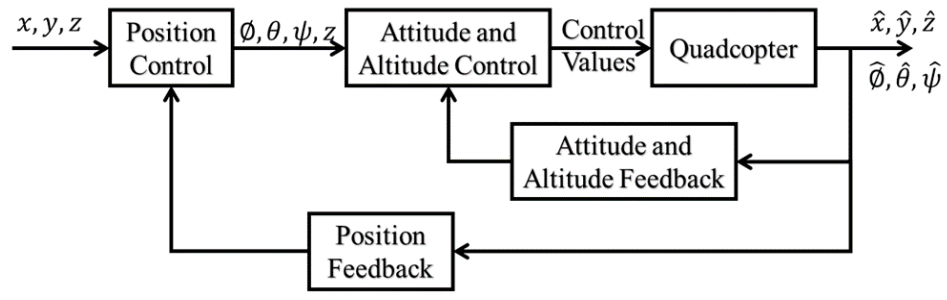

Figure 2 General block diagram of the quadcopter control system

The basic concept of the DIC scheme is to control the nonlinear system by training the inverse of the nonlinear system so that it cancels the system dynamics of the plant, which is ultimately the output response according to the reference. A simple representation of the general scheme of the DIC can be seen in Figure 3. The mathematical models can be written as in Equations 4 and 5, with $r_{(t)}$ being the reference, $y_{(t)}$ being the output, $f_{(t)}$ being the plant, and $f_{(t)}^{-1}$ being the plant inverse. The steps that must be taken to obtain a DIC scheme are identifying the plant and the inverse of the plant.

$$
\begin{gathered}
y_{(t)}=f_{(t)} \cdot f_{(t)}^{-1} \cdot r_{(t)} \\
y_{(t)} \cong r_{(t)}
\end{gathered}
$$

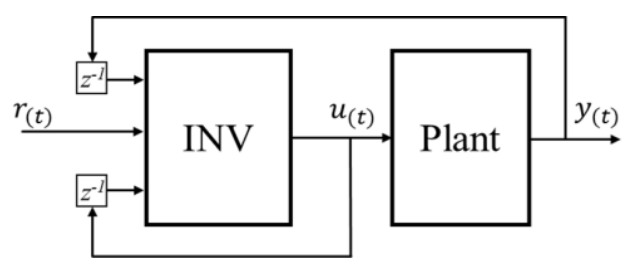

Figure 3 General direct inverse control scheme

Numerous methods have been developed for the identification of nonlinear systems. A method of nonlinear system modeling in a time series is the nonlinear autoregressive exogenous (NARX) model. NARX is a nonlinear autoregressive model that has exogenous input in time series modeling - that is, it has past values of the same series and current and past values of the driving series. Such a model can be expressed as:

$$
y_{(t)}=f\left[y_{(t-1)}, y_{(t-2)}, \ldots y_{(t-n)}, u_{(t)}, u_{(t-1)}, \ldots u_{(t-m)}\right]
$$

where $y_{(t)}$ is an output model, $u_{(t)}$ is an input model, $n$ is the maximum lag of input, and $m$ is the maximum lag of output. This NARX model is used for plant identification. Since the DIC scheme is the inverse of the plant obtained by Equation 4, the inverse is obtained as follows: 


$$
u_{(t)}=f^{-1}\left[y_{(t+1)}, y_{(t)}, \ldots y_{(t-n+1)}, u_{(t-1)}, u_{(t-2)}, \ldots u_{(t-m+1)}\right]
$$

Using the identification model in Equation 6 and the inverse model in Equation 7, the NN architecture schemes are obtained with the identification model (Figure $4 \mathrm{a}$ ) and the inverse model (Figure 4b). The artificial NN of the model identification used in this work consists of 20 input neurons. These inputs contain four motor velocities $\left(u_{(t)}\right)$, with onetime-delayed $\left(u_{(t-1)}\right)$ and two-time-delayed $\left(u_{(t-2)}\right)$ values, respectively, and one-timedelayed $\left(y_{(t-1)}\right)$ and two-time-delayed $\left(y_{(t-2)}\right)$ values of altitude and flight angles-that is, roll, pitch, and yaw. Then, hidden layer has 40 neurons and the output layer has 4 neurons, which are for roll, pitch, yaw, and altitude $\left(y_{(t)}\right)$.

The artificial NN of the inverse model also consists of 20 input neurons. These inputs are the entry points for the references of altitude and flight angles - that is, roll, pitch, and yaw $\left(y_{(t)}\right)$-together with each of their one-time-delayed $\left(y_{(t-1)}\right)$ and two-time-delayed $\left(y_{(t-2)}\right)$ values, respectively, and one-time-delayed $\left(u_{(t-1)}\right)$ and two-time-delayed $\left(u_{(t-2)}\right)$ values of the four motor velocities. Then, hidden layer has 50 neurons and the output layer has 4 neurons, which are the four motor velocities $\left(u_{(t)}\right)$.

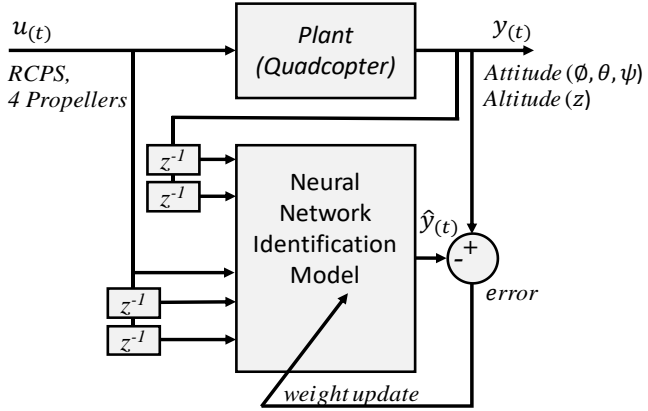

(a)

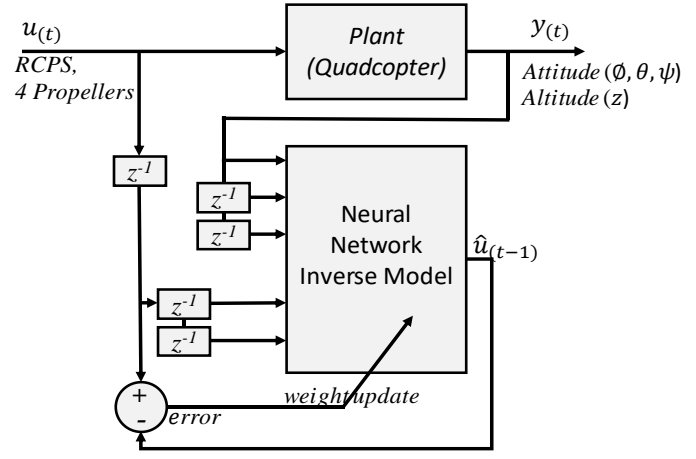

(b)

Figure 4 (a) Identification model and (b) inverse model neural networks

\subsection{Data Acquisition}

As previously noted, the focus of this research was on developing an attitude and altitude control system that can effectively handle maneuvering conditions. To obtain a model that closely simulated the real quadcopter, the NN was trained on real flight data with considerable variations in altitude, hover, and especially maneuvers. To validate the capabilities of the system obtained from the training results, testing data were inputted into the NN-DIC system again as simulation results and compared with the real data to calculate the deviation error.

To obtain real flight data for training and testing, this study used the quadcopter developed in the Computational Intelligence and Intelligent Systems Laboratory, Universitas Indonesia. The total weight of the developed quadcopter is $1.2 \mathrm{~kg}$. As an actuator, this quadcopter uses a combination of a T-Motor $940 \mathrm{KV}-\mathrm{BLDC}$ and $12^{\prime \prime} \times 5^{\prime \prime}$ carbon propellers, with a T-Motor 18 A electronic speed control placed at each corner. The quadcopter is powered by a $14.8 \mathrm{~V}$ lithium polymer battery. This configuration can produce an ideal thrust of $2.6 \mathrm{~kg}$, so the quadcopter can take off and maneuver easily. Ardupilot 2.5 is used as the flight control unit (FCU), and the PID method is used as the main control. Ardupilot 2.5 is an open-source FCU consisting of a microcontroller and inertial measurement units, such as a gyroscope, an accelerometer, and a barometer. 


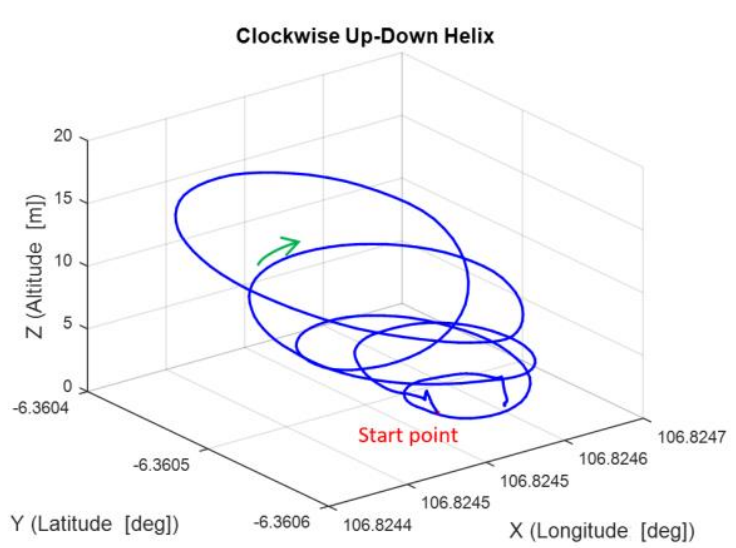

(a)

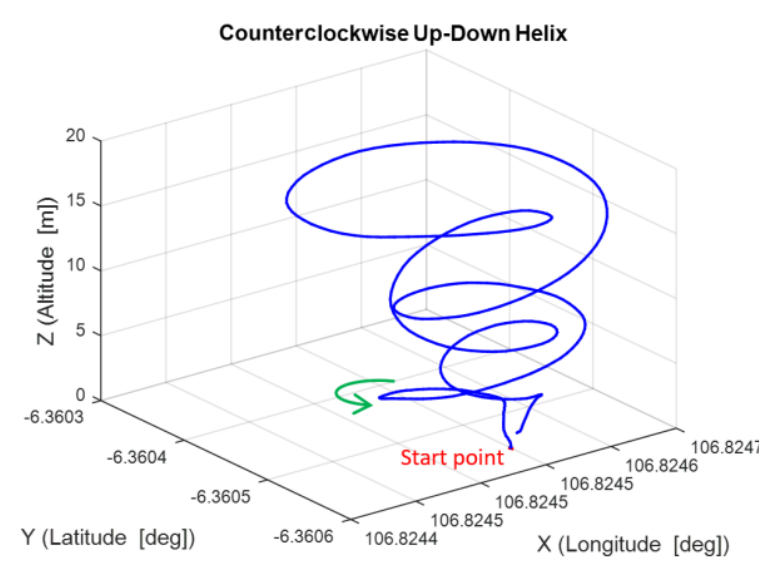

(c)
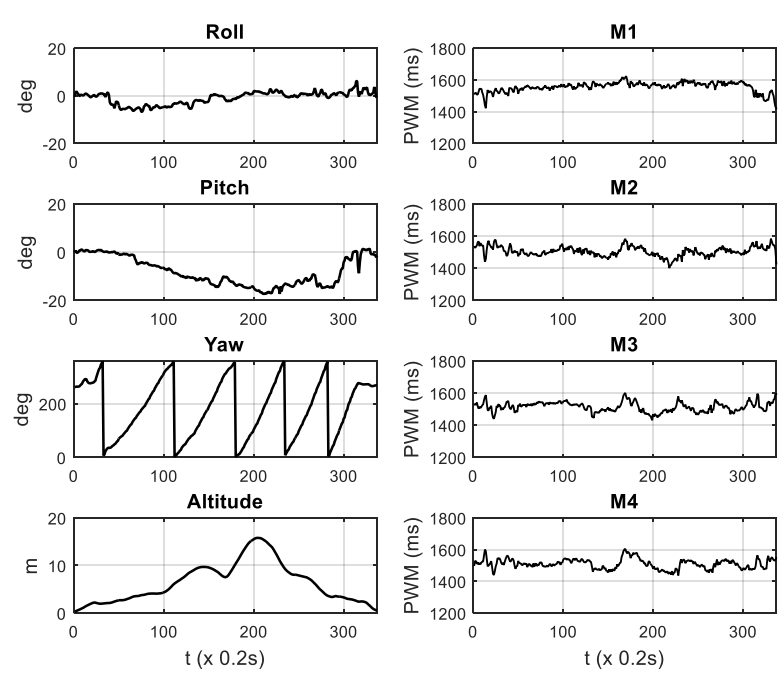

(b)
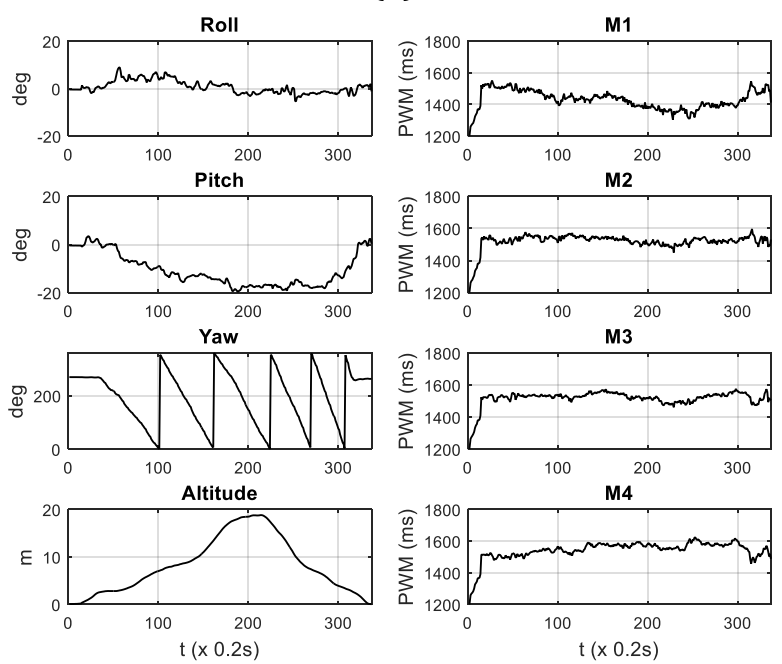

(d)

Figure 5 Training datasets: (a) Three-dimensional plot of clockwise up-and-down helix movement; (b) Attitude, altitude, and pulse-width modulation of propellers on clockwise up-and-down helix movement; (c) Three-dimensional plot of counterclockwise up-and-down helix movement; and (d) Attitude, altitude, and pulse-width modulation of propellers on counterclockwise up-and-down helix movement

The training dataset is shown in Figure 5, and the testing dataset is shown in Figure 6. Since the quadcopter used in this research is operated by a pilot, its flight was conditioned on a scenario that met the research goals. In this scenario, the quadcopter made a vertical takeoff and continued with an up-and-down helix maneuver in both clockwise and counterclockwise motions.

Figure 5a shows the clockwise up-and-down helix movement, and Figure $5 b$ shows the experimental real flight data of attitude, altitude, and PWM of each propeller. Figure 5c shows the counterclockwise up-and-down helix movement, and Figure $5 \mathrm{~d}$ shows the experimental data of attitude, altitude, and PWM of each propeller. As shown in Figure 5, the experimental real flight data varied greatly. These data were expected to be sufficient for identification and inverse modeling according to the real quadcopter conditions using an NN. Similarly, the testing data also included various movements (Figure 6). 


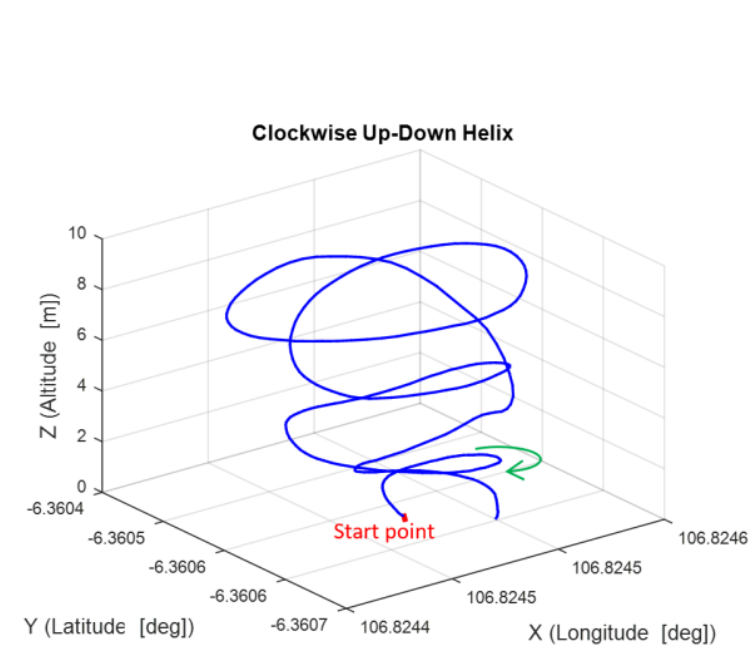

(a)

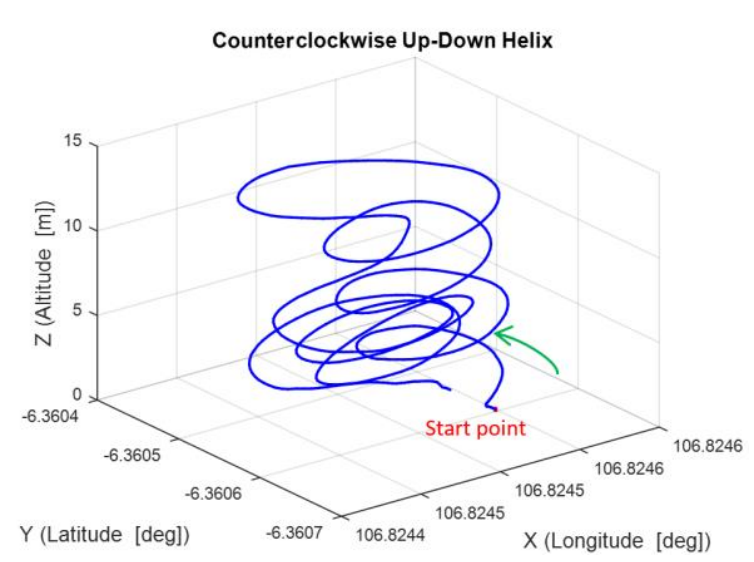

(c)
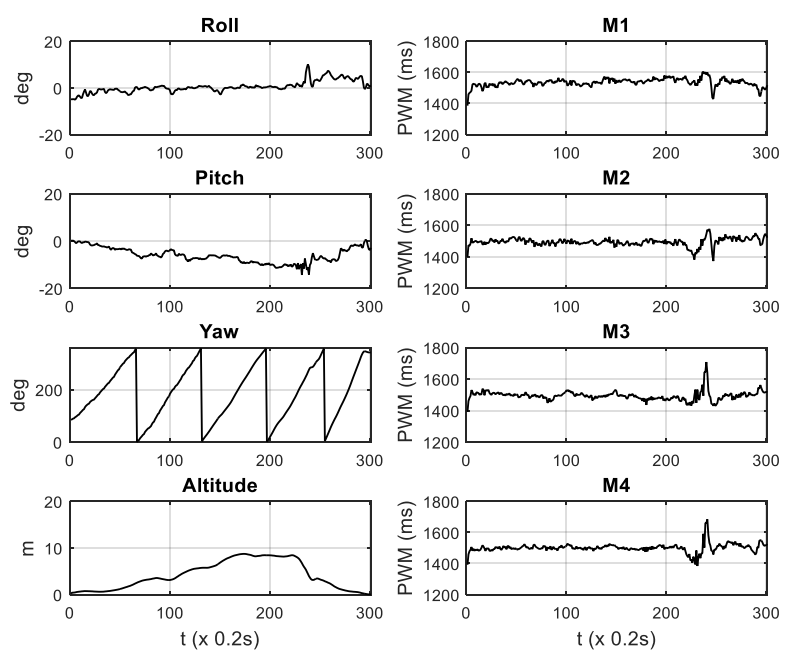

(b)
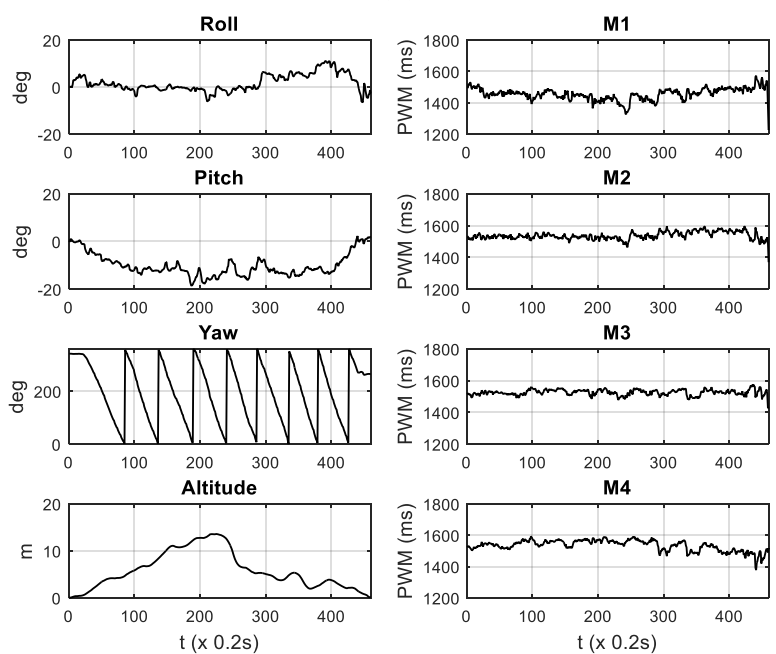

(d)

Figure 6 Testing dataset: (a) Three-dimensional plot of clockwise up-and-down helix movement; (b) Attitude, altitude, and pulse-width modulation of propellers on clockwise up-and-down helix movement; (c) Three-dimensional plot of counterclockwise up-and-down helix movement; and (d) Attitude, altitude, and pulse-width modulation of propellers on counterclockwise up-and-down helix movement

\section{Results and Discussion}

\subsection{NN-DIC System Performance in the Testing Dataset}

The performance of the NN-DIC system was evaluated using a testing dataset with characteristics similar to those of the training dataset to ensure that it could respond to changes in attitude and at the same time maintain the quadcopter's maneuvers in the trajectory. As previously explained, the four basic motions of the quadcopter are roll, pitch, yaw, and altitude. Roll and pitch affect maneuvers. Therefore, it is necessary to observe the performance of the NN-DIC system on attitude.

Figure 7 shows the test results. The overall mean squared error (MSE) between the testing dataset and the experimental results was 1.461. The MSE for roll was 3.104, the MSE for pitch was 0.889 , the MSE for yaw was 1.834 , and the MSE for altitude was 0.018 . 


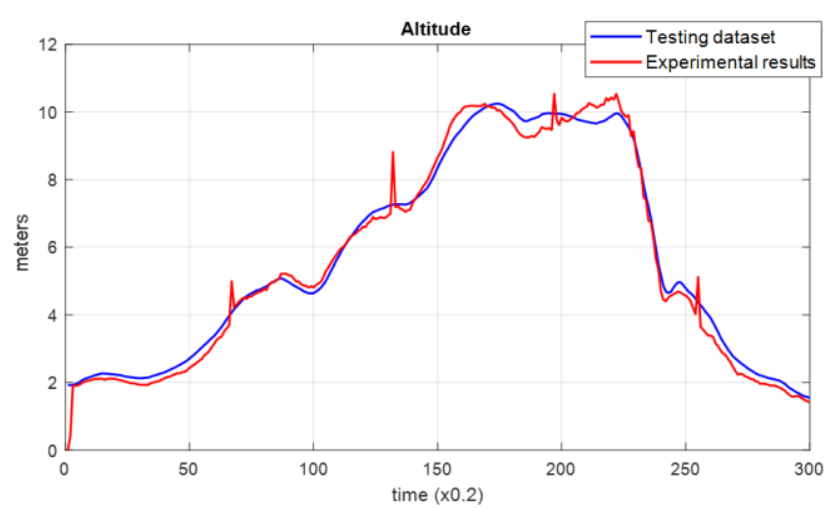

(a)
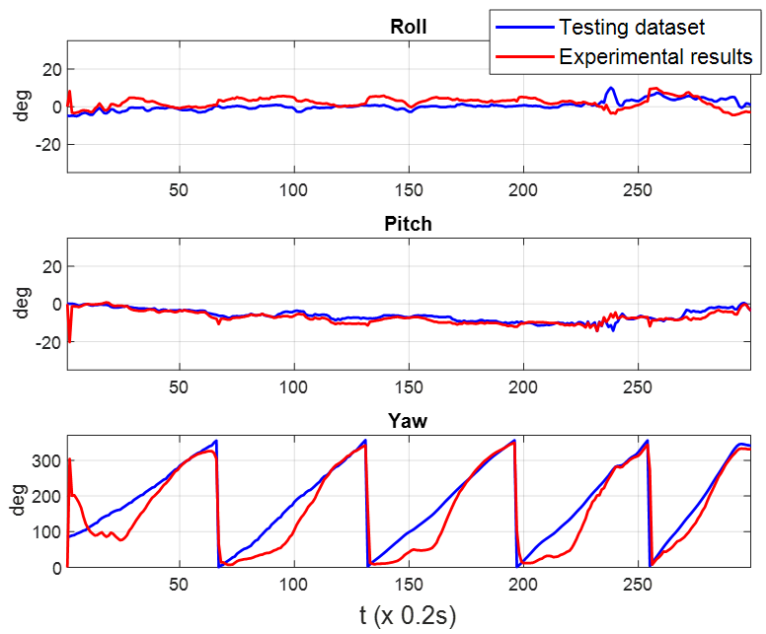

(b)

Figure 7 Neural network-based direct inverse control simulation results: (a) Attitude; and (b) Altitude

As shown in Figure 7, the NN-DIC system was overall able to follow the trajectory. The attitude and altitude were maintained following the trajectory, with small MSEs for attitude, roll, and pitch. In Figure 7a, it can be seen that the altitude control worked well, following the trajectory. During takeoff, the quadcopter followed the trajectory to its highest altitude. In Figure $7 b$, it can be seen that deviations occurred in roll, pitch, and yaw, showing the controller's attempt to correct its vertical error at the ramp, rising to the altitude reference.

Figure 8 (altitude) shows that the quadcopter followed the trajectory until the 13th second, when the simulation exhibited overshoot. This occurred due to an extreme change in the yaw angle from $360^{\circ}$ to $0^{\circ}$. Figure 8 (yaw) shows that in real flight, the quadcopter's direction did not change significantly. In the next stage, the NN-DIC system responded so that the quadcopter could follow the trajectory.

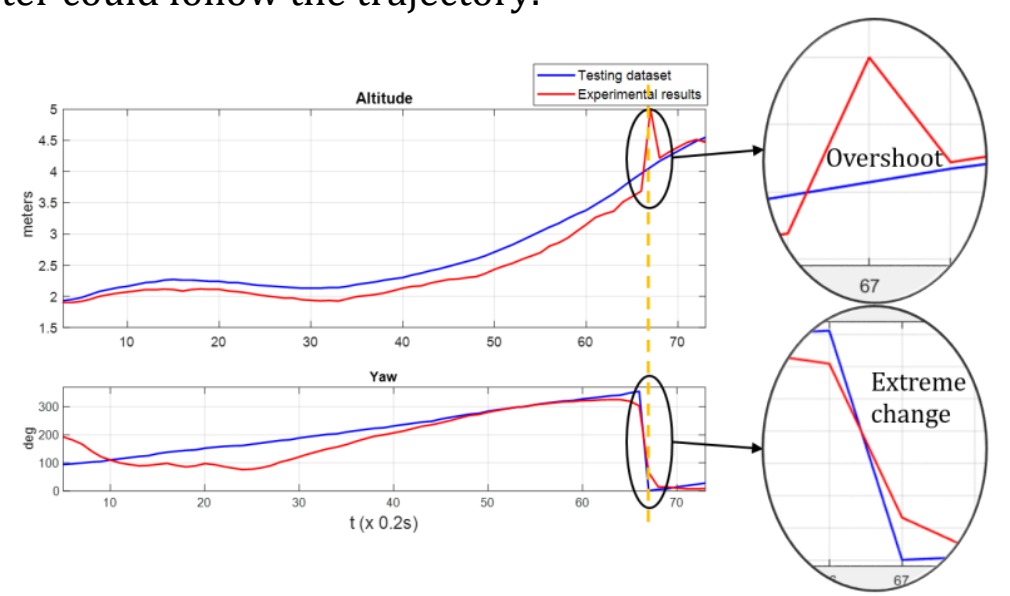

Figure 8 Altitude overshoot caused by an extreme change in the yaw angle

\subsection{NN-DIC System Performance on Ramp Trajectory Altitude}

The experiment was continued by providing a trajectory. The objective of this experiment was to observe the performance of the NN-DIC system on a given ramp trajectory. The ramp altitude trajectory maneuver was chosen because it comprises the four basic motions of the quadcopter. Figure 9 shows that the NN-DIC system was able to follow the trajectory. The ripple along the ramp reference line indicates that the NN-DIC system made active corrections to roll, pitch, and yaw to increase the altitude. This is natural, as 
small quadcopters are usually not sufficiently balanced to make center gravity at the center of all four rotors. This small imbalance interacts with the significant nonlinearity and the strength of the cross-coupling between the quadcopter's modes of motion. Thus, with every small change in altitude, the quadcopter also tries to maintain its equilibrium to continue increasing its altitude.

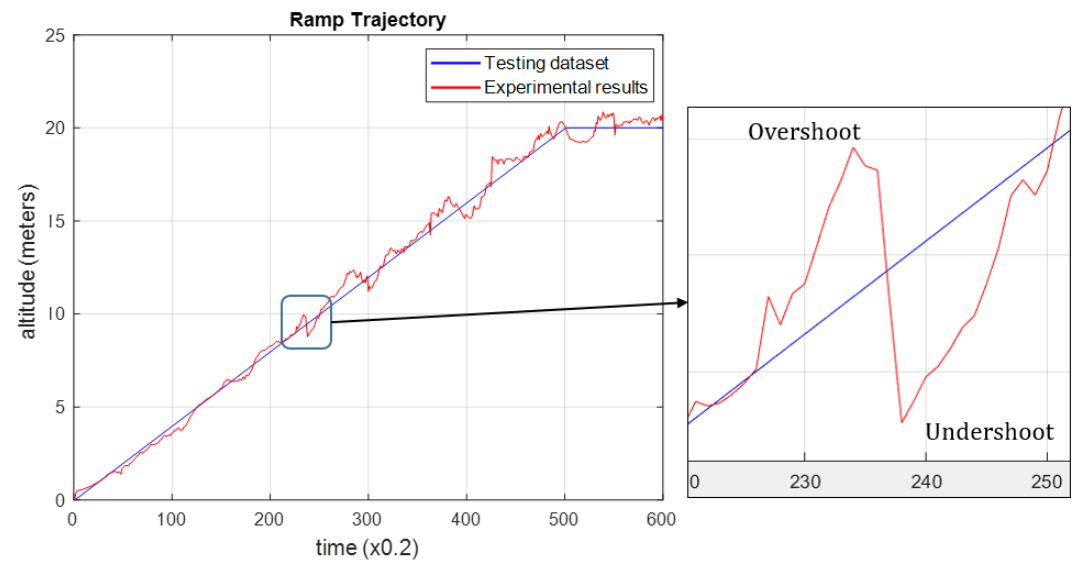

Figure 9 Experimental results of the altitude ramp trajectory

The quadcopter attempted to increase its altitude and experienced overshoot. Subsequently, it quickly reduced its altitude, thus obtaining its reference altitude. However, the reduction in the previous altitude caused the altitude to undershoot and the quadcopter to rise again rapidly. This cycle lasted approximately $5 \mathrm{~s}$ along the ramp route.

\section{Conclusions}

The purpose of this study was to continue our research on exploring the capabilities of NN-DIC applied to a quadcopter under maneuver conditions. The NN-DIC system succeeded in maintaining the quadcopter's trajectory. The experimental results also showed that the quadcopter could follow the trajectory altitude with a very small MSE (0.018), as well as some inaccuracy in attitude. These results indicate that the NN-DIC system can control a quadcopter under maneuver conditions, such as clockwise/counterclockwise up-and-down helix movements. A full quadcopter control system is being developed and will be presented in the near future.

\section{Acknowledgements}

The authors would like to express their gratitude to the Ministry of Research, Technology, and Higher Education of Indonesia for supporting this work through a Universitas Indonesia Scholarship Research Grant number 1251.38/E4.4/2012. We also thank Universitas Dian Nuswantoro.

\section{References}

Alkamachi, A., Erçelebi, E., 2017. Modelling and Genetic Algorithm Based-PID Control of HShaped Racing Quadcopter. Arabian Journal for Science and Engineering, Volume 42(7), pp. 2777-2786

Anuradha, D.B., Reddy, G.P., Murthy, J.S.N., 2009. Direct Inverse Neural Network Control of a Continuous Stirred Tank Reactor (CSTR). In: Proceedings of the International MultiConference of Engineers and Computer Scientists, Volume 3

Argentim, L.M. Rezende, W.C., Santos, P.E., Aguiar, R.A., 2013. PID, LQR and LQR-PID on a 
Quadcopter Platform. In: 2013 International Conference on Informatics, Electronics and Vision (ICIEV), pp. 1-6

Bresciani, T., 2008. Modelling, Identification and Control of a Quadrotor Helicopter. Master's Thesis, Graduate Program, Lund University, Sweden

Duan, H., Shao, S., Su, B., Zhang, L., 2010. New Development Thoughts on the Bio-inspired Intelligence Based Control for Unmanned Combat Aerial Vehicle. Science China Technological Sciences, Volume 53(8), pp. 2025-2031

Gandhi, D.A., Ghosal, M., 2018. Novel Low Cost Quadcopter for Surveillance Application. In: 2018 International Conference on Inventive Research in Computing Applications (ICIRCA), pp. 412-414

Gheorghita, D., Vîntu, I., Mirea, L., Brăescu, C., 2015. Quadcopter Control System. In: 2015 $19^{\text {th }}$ International Conference on System Theory, Control and Computing, ICSTCC 2015, pp. 421-426

Heryanto, M.A., Suprijono, H., Suprapto, B.Y., Kusumoputro, B., 2017. Attitude and Altitude Control of a Quadcopter using Neural Network Based Direct Inverse Control Scheme. Advanced Science Letters, Volume 23(5), pp. 4060-4064

Heryanto, M.A., Wahab, W., Kusumoputro, B., 2015. Optimization of a Neural Network Based Direct Inverse Control for Controlling a Quadrotor Unmanned Aerial Vehicle. MATEC Web of Conferences, Volume 34, pp. 4-7

Krishnan, R.A., Jisha, V.R., Gokulnath, K., 2018. Path Planning of an Autonomous Quadcopter Based Delivery System. In: 2018 International Conference on Emerging Trends and Innovations in Engineering and Technological Research (ICETIETR), pp. 1-5

Mahadika, P., Subiantoro, A., Kusumoputro, B., 2020. Neural Network Predictive Control Approach Design for Adaptive Cruise Control. International Journal of Technology, Volume 11(7), pp. 1451-1462

Muliadi, J., Kusumoputro, B., 2018. Neural Network Control System of UAV Altitude Dynamics and its Comparison with the PID Control System. Journal of Advanced Transportation, Volume 2018, Article ID 3823201

Najm, A.A., Ibraheem K.I., Ahmad T.A., Amjad J.H., 2020. Genetic Optimization-Based Consensus Control of Multi-Agent 6-DoF UAV System. Sensors, Volume 20(12), pp. 131

Najm, A.A., Ibraheem, I.K., 2019. Nonlinear PID Controller Design for a 6-DOF UAV Quadrotor System. Engineering Science and Technology, an International Journal, Volume 22(4), pp. 1087-1097

Nenni, M.E., Di Pasquale, V., Miranda, S., Riemma, S., 2020. Development of a DroneSupported Emergency Medical Service. International Journal of Technology, Volume 11(4), pp. 656-666

Nguyen, A.T., Xuan-Mung, N., Hong, S.-K., 2019. Quadcopter Adaptive Trajectory Tracking Control: A New Approach via Backstepping Technique. Applied Sciences, Volume 9(18), pp. 2-17

Suprapto, B.Y., Mustaqim, A., Wahab, W., Kusumoputro, B., 2017. Modified Elman Recurrent Neural Network for Attitude and Altitude Control of Heavy-Lift Hexacopter. In: 2017 $15^{\text {th }}$ International Conference on Quality in Research (QiR): International Symposium on Electrical and Computer Engineering, pp. 309-314

Tripathi, V.K., Behera, L., Verma, N., 2015. Design of Sliding Mode and Backstepping Controllers for a Quadcopter. In: 2015 39th National Systems Conference, pp. 1-6

Wang, P., Man, Z., Cao, Z., Zheng, J., and Zhao, Y., 2016. Dynamics Modelling and Linear Control of Quadcopter. In: 2016 International Conference on Advanced Mechatronic Systems (ICAMechS), pp. 498-503 
Xianglei, D., Shuguang, Z., Lvchang, H., and Rong, H., 2011. The Neural Network Direct Inverse Control of Four-Wheel Steering System. In: 2011 Third International Conference on Measuring Technology and Mechatronics Automation, pp. 865-869

Yuning, J., Rasool, M.A.U., Bo, Q., Farid, G., and Chaudary, S.T., 2019. An Adaptive Neural Network State Estimator for Quadrotor Unmanned Air Vehicle. In: (IJACSA) International Journal of Advanced Computer Science and Applications, Volume 10(2), pp. 316-321 